\title{
Labyrinthe
}

25 | 2006 (3)

La Bande dessinée : ce qu'elle dit, ce qu'elle montre

\section{Extérieur nuit}

Notes autour de quelques récits courts de Gipi

\section{Valerio Rota}

\section{(2) OpenEdition}

Journals

Édition électronique

URL : http://journals.openedition.org/labyrinthe/1413

DOI : $10.4000 /$ labyrinthe. 1413

ISSN : 1950-6031

Éditeur

Hermann

\section{Édition imprimée}

Date de publication : 1 décembre 2006

Pagination : 61-67

ISBN : 2-9526131-2-5

Référence électronique

Valerio Rota, «Extérieur nuit », Labyrinthe [En ligne], 25 | 2006 (3), mis en ligne le 28 mars 2010, consulté le 21 avril 2019. URL : http://journals.openedition.org/labyrinthe/1413 ; DOI : 10.4000/ labyrinthe.1413

Ce document a été généré automatiquement le 21 avril 2019

Propriété intellectuelle 


\title{
Extérieur nuit
}

\author{
Notes autour de quelques récits courts de Gipi
}

\section{Valerio Rota}

1 On continue à parler beaucoup de Gipi, surtout après qu'il a remporté à Angoulême le prix du meilleur album de l'année 2005 pour Notes pour une histoire de guerre. Son succès provient peut-être du fragileéquilibrequ'il parvient à instaurer: ses histoires sont indéniablement "italiennes », mais elles possèdent une portée qui dépasse de beaucoup le cadre national; elles sont situées dans le présent, mais leur vraie dimension est intemporelle; enfin, le caractère fortement autobiographique des récits de Gipi n'empêche pas que les personnages et les situations représentés soient étrangement familiers au lecteur. Ce dernier fait ainsi siennes les expériences singulières racontées et dessinées par Gipi, auteur à la fois personnel et universel.

2 Extérieur nuit est un exemple remarquable de cette esthétique : ils'agit d'un recueil de récits courts parus en Italie chez Coconino Press en 2003, puis réimprimés en 2005, dans une édition enrichie d'un récit supplémentaire et d'un coloriage révisé. L'objet est singulier: plusieurs gradations de bleu sont la seule couleur employée; les traits noirs, très minces, sont rares, et utilisés pour des effets stylistiques et narratifs spécifiques l'usage du seul trait noir, sans coloriage, introduit un autre niveau de la narration, parfois onirique ou visionnaire. Le titre, emprunté au langage du cinéma, n'est pas mensonger : le lecteur découvre une œuvre composée de planches obscurcies; les scènes semblent toutes "tournées" la nuit; la lumière si particulière est obtenue par "manque ", en égratignant la couleur en excès. De fait, ce qui est effacé semble avoir la même importance que ce qui ne l'est pas : on le voit très bien, par exemple, dans la treizième planche de « Muttererde ", le dernier récit du livre, où un long texte écrit à la main est parcouru par un mot transversal («FOUTAISES ») qui le neutralise et le dévalorise, mais il reste encore entièrement lisible. Gipi évolue toujours dans cet espace incertain entre le dit et le non-dit, entre l'à peine visible et le quasi effacé.

3 Une introduction textuelle au volume informe le lecteur que «La Tronche ", premier récit du recueil, trouve son origine dans l'angoisse suivant les attentats du 11 septembre 2001. Une tour frappée par un avion est dessinée dans la première planche; mais, au début de la deuxième planche, on assiste à l'intrusion d'un personnage étrange, le 
mystérieux La Tronche, un homme aux traits irréguliers, qui exige que l'on raconte son histoire au lieu de celle des attentats. L'angoisse universelle pour le futur de l'humanité fait donc place à une historiette de province, un petit fait divers; le plan général sur le grand édifice de l'Histoire se rétrécit et l'objectif se focalise sur une des briques qui le composent, une restriction de champ littéralement imposée à l'auteur par un personnage. L'alternance des techniques (huile, encre, puis encore huile) permet d'obtenir un triple plan de narration: le plan qui, au début, montre l'attentat aux tours, et qui demeure isolé ; un deuxième plan, dont les cases sont réalisées seulement par un trait d'encre noir, sans couleur, représente la voix de La Tronche qui hante l'auteur et qui impose son histoire ; finalement, un troisième plan, réalisé à l'huile, raconte l'histoire de La Tronche. Néanmoins, ces dimensions semblent ne pas être complètement distinctes: les deux techniques envahissent réciproquement les plans qu'elles racontent. C'est, encore une fois, La Tronche lui-même qui semble suggérer à l'auteur la technique qu'il doit utiliser pour raconter son histoire: dans la deuxième planche, des voyous tracent des dessins obscènes en écorchant les parois noircies d'une galerie incrustées d'oxyde de carbone, tout comme Gipi érafle la feuille pour enlever la couleur excédante. Au cœur du récit, un moment de crise : les voyous harcèlent un vigile, qui, excédé, sort son pistolet et les met en joue; c'est La Tronche qui va sauver les jeunes loubards en rossant le vigile. Son intervention correspond à une soudaine prise de pouvoir du texte sur l'image, reléguée au second plan. Manière de fixer l'apogée de la tension dans un espace déréglé où règne la violence : le monde extérieur paraît bien pire que la prison, où La Tronche est respecté et traité en ami. L'histoire se termine avec un renversement complet qui ramène La Tronche dans la normalité, représentée du point de vue symbolique et narratif par son mariage et par sa mort « conventionnelle » (due à un arrêt cardiaque) et, du point de vue graphique, par les visages « parfaitement ronds » de ses deux enfants, tandis qu'on devine à l'arrièreplan le cimetière où leur père est enterré...

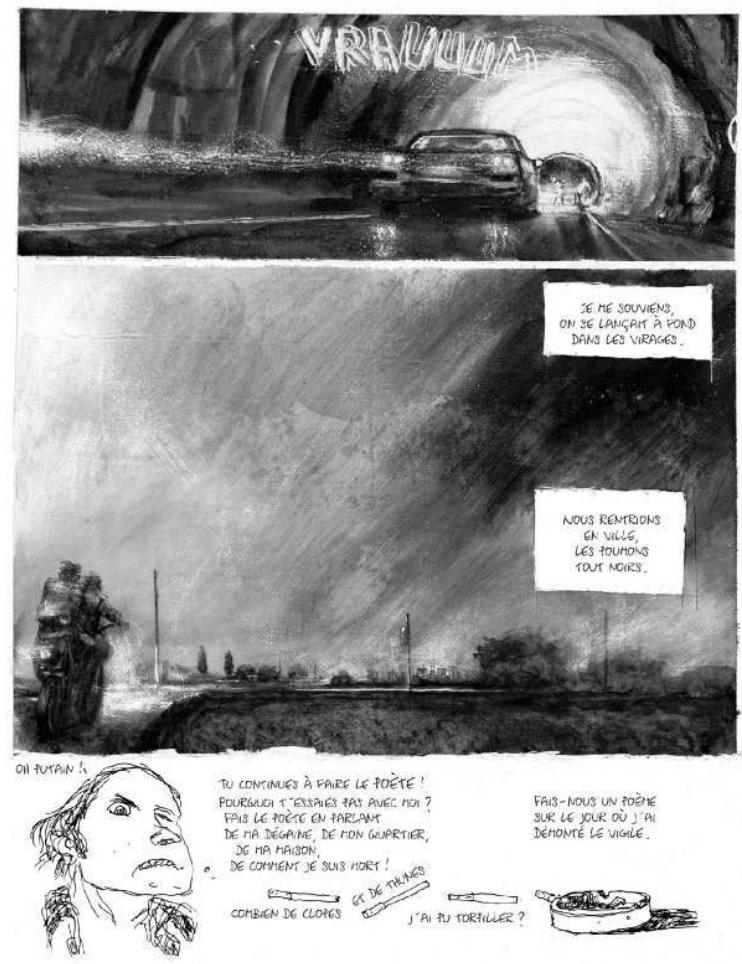

CGIPI, COCONINO PRESS \& VERTIgE GRAPHIC, 2005. 
Si l'histoire de La Tronche glissait d'un dérèglement initial à une (triste) normalité retrouvée, la démarche est renversée dans le récit suivant, où l'on va de la normalité au bouleversement - celui-ci restant cependant à l'horizon du récit. Dans «Rue des Lauriers ", en effet, un épisode troublant ${ }^{1}$ est raconté par son absence même: le récit, très statique, évoque par petites touches une enfance "sans histoire ", manière de dépeindre l'état des choses avant qu'une « rupture » ne se produise (celle, matérielle, d'une fenêtre, qui est aussi celle d'un état de tranquillité, un moment de bouleversement dans la routine d'une famille). C'est une façon d'appréhender la rupture elle-même, et de mesurer son poids spécifique, sans l'expliquer ou l'analyser: montrer la situation qui va être bouleversée, c'est évidemment faire éprouver ce que la rupture signifie réellement, et le traumatisme dont elle sera cause. L'événement négatif - un intrus pénètre dans la chambre des enfants : agression? viol ? On ne le saura pas - qui jamais ne se manifeste au cours de la narration, sinon dans la dernière case, et encore, très allusivement, est donc présent "en creux»; et, même à la fin, on essaiera d'expliquer l'anomalie par une justification rassurante (le bruit suspect de la fenêtre brisée sera comparé au bruit d'une cuillère qui tombe sur le sol). Des textes, éraflés sur la couleur du dessin, et qui représentent peut-être les arrière-pensées de l'enfant reconstruites à travers le souvenir, sont parfois presque inintelligibles; et la calligraphie, en plusieurs endroits, se fait nerveuse. Mais, quand intervient la "rupture", dans la dernière case, ce sont les caractères d'une machine à écrire qui sont employés : tentative d'effacer, au moins de rationaliser tout désordre, même celui, inoffensif, du quotidien (sensible non seulement dans ces textes "sauvages", mais aussi, par exemple, dans l'épisode des jouets laissés hors de la porte le soir et retrouvés, le lendemain, là où on les avait laissés) à travers l'utilisation d'une graphie régulière et mécanique, qui nie le choc de l'événement négatif en introduisant une «réglementation». Manière aussi, par cette typographie journalistique, également celle du procès-verbal tapé à la machine au commissariat, de faire glisser l'événement dans l'ordre du fait divers, banal, et de l'anonyme. Les pages qui précèdent retournent pourtant l'effet de ces lignes, car cette écriture mécanique finale est aussi la manifestation de la « rupture » : où le désordre se dévoile à travers l'ordre.

5 Dans le récit suivant, le désordre règne. L'époque où se déroulent les événements des «Visages dans l'eau » semble imaginaire, ou du moins non identifiable: des références à des bombardements et à une guerre encours en Italie semblent placer l'action dans un passé peu défini; mais des références à l'acteur pornographique Rocco Siffredi, qui situent le récit dans le présent, provoquent une sorte de court-circuit chronologique. Deux amis et une jeune fille essaient d'échapper à on ne sait quoi; ou, mieux, on comprend que la fille cherche à fuir un passé de prostitution ainsi que ses protecteurs. La maladie mentale - là encore, supposée - de l'un des deux, transformée en élément fantastique (les « visages dans l'eau » que le protagoniste croit voir appartiennent, selon lui, à des gens morts sous les bombardements, et il croit pouvoir parler avec eux), semble être la clef de la narration: les deux sous-fifres musclés venus pour rattraper la fille disparaîtront physiquement, d'une manière très mystérieuse, au moment précis où ils semblent avoir triomphé. Cette disparition ne sera expliquée que par l'aide des « visages dans l'eau » : c'est que la voix narrative et le point de vue principal du récit appartiennent au personnage fou. Les mots des autres personnages laissent entendre qu'en réalité il peut aussi y avoir d'autres explications, moins surnaturelles, mais le lecteur reste en suspens, plongé dans un monde sombre où l'orage est toujours au bord d'éclater. Les textes, qui dans les autres récits semblaient « ronger » les dessins en les éraflant et en les 
envahissant, sont ici plus disciplinés. Le domaine du texte parait être canoniquement confiné aux récitatifs et aux bulles ; à l'intérieur du dessin, la communication textuelle est effacée (neuvième planche, deuxième case), ou timidement utilisée en syllabes discrètes à des fins banalement onomatopéiques («PO», par exemple, pour indiquer le bruit d'un moteur).

Paradoxalement, le code textuel sera utilisé seulement au moment où il faudra communiquer avec les fantasmatiques "visages dans l'eau», c'est-à-dire quand on s'attend à l'emploi de moyens moins rationnels et plus instinctifs. La connaissance est donc éminemment graphique, visuelle; les couleurs sont très sombres, la lumière est presque absente, le ciel souvent noir, comme si l'auteur voulait souligner les troubles de perception qui affectent les personnages (du moins certains d'entre eux), et les faire partager au lecteur.

7 Si dans « Les visages dans l'eau » la maladie mentale est l'élément perturbant, qui distord la réalité, dans «Voiture sous la pluie » la confusion est produite par une duplication des niveaux narratifs : on assiste ici à une sorte de situation méta-cinématographique, où l'un des personnages croit être le protagoniste d'un film diffusé sur la chaîne italienne Retequattro. Celui-ci ne cesse de commenter, en spectateur, les événements dont il est l'acteur. Encore une fois, comme dans «La Tronche», l'emploi du trait d'encre sans couleur (troisième planche) semble exprimer un plan différent, intérieur (les pensées de l'un des personnages: un "acteur " répète mentalement les actions qu'il va «interpréter »). Le récit met en scène des trafiquants de drogue au moment d'une transaction : une situation hautement codifiée, où chaque geste (attendre la voiture des vendeurs, descendre de la voiture, s'approcher du vendeur...) doit être accompli suivant un protocole strict et rigoureux. Le parallèle avec un contexte théâtral s'impose de luimême. Le scénario demeure cependant peu clair pour les autres personnages ; le probable metteur en scène, celui qui dirige les autres "acteurs", est un mafieux illettré (en donnant des ordres, il confondra le verbe italien abbagliare, éblouir, avec le mot abbaiare, aboyer, poussant son subordonné à aboyer pour de vrai ${ }^{2}$ ) ; les phares d'une voiture font office de projecteurs pour éclairer le lieu de l'action. Mais le vrai réalisateur est l'auteur Gipi, qui, seul, a le pouvoir d'écrire le mot « fin » en bas de la dernière page.

8 «Les cinq virages » met en évidence deux autres éléments récurrents dans les récits de Gipi : le regard enfantin et la co-existence d'éléments anachroniques. Les souvenirs sont reconstruits dans le présent de l'âge adulte, mais ils revivent tout en gardant la focalisation de l'enfant qui les a vécus en son temps. Après la première planche, qui se déroule manifestement dans le passé, le récit emploie abruptement le présent de l'indicatif; faut-il y voir un réel changement temporel dans la narration? On ne sait s'il faut considérer la première planche (au passé) comme une introduction détachée du reste du récit (au présent), ou si les deux moments narratifs sont contemporains. Des éléments chronologiquement incongrus (tout comme dans «Les visages dans l'eau ») contribuent à brouiller un peu plus les idées du lecteur: dans la même planche $\left(n^{\circ} 5\right)$, on trouve des références à Valentino Rossi et Loris Capirossi, champions de motocyclisme d'aujourd'hui, et aux Twin Towers qui tremblent, comparées à des bouteilles de verre agitées par des vibrations ; mais on trouve aussi, à plusieurs reprises, un élément visuel anachronique: le maillot de la Juventus de Turin apparaît décoré d'une seule étoile, signalant que l'équipe n'a pas encore gagné son vingtième championnat, donc qu'on est dans une époque antérieure à 1982. Extérieur nuit représente ainsi, dans la pluralitéde ces courts récits, une tentative,jamais pleinement achevée, toujours au bord de l'échec, 
d'utiliser la narration en Bande dessinée comme moyen d'explorer les strates les plus imperceptibles de la réalité. Les personnages principaux des histoires de Gipi semblent tous avoir le même défaut: ils n'arrivent pas à déchiffrer les événements qui se produisent autour d'eux, la chaîne des causes et des effets leur échappe, enfants, fous, ou simplement perturbés par l'irruption de l'inexplicable, la raison est pour eux sans secours, et le seul moyen pour eux d'atteindre un état plus lucide de conscience, c'est de raconter, de confier leurs pensées et leurs souvenirs à la narration. La réalité du personnage elle-même peut être mise en discussion: c'est le cas de La Tronche, qui s'attache à garantir son existence en stimulant la production d'une narration. Qu'il s'agisse de souvenirs d'enfance ou de fiction pure, Gipi, dans (et par) ses narrations graphiques, se propose donc une tâche: comprendre le monde sans chercher à l'expliquer, mais en l'effleurant, en grattant sa surface, très légèrement, pour tenter d'y saisir quelques brins de réalité.

\section{NOTES}

1. Il resterait d'ailleurs bien mystérieux sans l'évocation, là encore allusive, qu'en fait Gipi dans l'introduction.

2. Le jeu de mots est rendu en français par « ablouie-leur ».

\section{AUTEUR}

\section{VALERIO ROTA}

valerio.rota[at]gmail.com 